\title{
Experimental study of anisotropic constitutive behavior of $\beta-H M X$ crystals via nanoindentation and small-scale dynamic impact
}

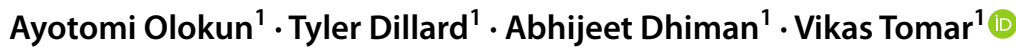

Received: 14 August 2021 / Accepted: 2 November 2021

Published online: 17 November 2021

(c) The Author(s) 2021 OPEN

\begin{abstract}
For energetic crystals such as HMX, the sensitivity of the material to shock, the possibility of initiation, and the subsequent reaction is known to be controlled by processes occurring at the crystal level. The anisotropic nature of $\beta$ - $H M X$ can be critical in determining the performance of $\mathrm{HMX}$ based polymer bonded explosives, which are widely used across multiple industries as propellant or explosives. In this work, we experimentally obtain constitutive parameters for characterizing the response of multiple crystalline planes of $\beta-H M X$ crystals to external loading. Nanoindentation and smallscale dynamic impact experiments were performed on multiple planes of $\beta-\mathrm{HMX}$ crystals to comparatively measure the indentation moduli in multiple orientation directions. Anisotropic material behavior, involving constitutive elastic and non-elastic parameters, was measured and studied. Findings regarding material properties for the (100), (010), (001), $\{110\}$, and $\{011\}$ planes of $\beta-H M X$ are presented and compared with literature data.
\end{abstract}

Keywords HMX · Energetic materials · Anisotropy · Nanoindentation · Hardness

\section{Introduction}

Accurate characterization and prediction of the mechanical behavior of energetic materials (EMs) requires indepth understanding of their physical properties and of their response to external stimuli. There are many material responses whereby it is important to understand non-initiating behaviors, including deformation and failure, as damage may alter the safety parameters of these explosive material and set a new initial condition for future performance [1]. For an energetic material such as $\mathrm{HMX}$, sensitivity to shock, the possibility of initiation, and the subsequent reaction is known to be controlled by processes occurring at the crystal level. HMX occurs in four polymorphic forms, $a, \beta, \delta$ and $\varepsilon$. Of these, the most thermodynamically stable phase is $\beta-H M X$ [2]. Thermal stability of the $\beta-\mathrm{HMX}$ crystalline phase makes it a good candidate to characterize effect of anisotropy on $\mathrm{HMX}$ material behavior. Previous experiments have pointed to the anisotropic nature of $\beta-\mathrm{HMX}$ being critical in determining the performance of $\mathrm{HMX}$ based polymer bonded explosives (PBX) [3]. The main failure mechanisms in such materials have been identified as particle fracture, interfacial failure between particle and binder, and interfacial failure between particles [4]. With respect to particle fracture, due to the anisotropic nature of $\mathrm{HMX}$, it is known that deformation in single crystals of $\beta-H M X$ is orientation dependent and fracture tends to occur along cleavage planes. For instance, indentation experiments performed by Palmer and Field [5] on cleaved crystals determined the

Supplementary Information The online version contains supplementary material available at https://doi.org/10.1007/s42452-02104862-4.

$\triangle$ Vikas Tomar, tomar@purdue.edu; Ayotomi Olokun, aolokun@purdue.edu; Tyler Dillard, tdillard@purdue.edu; Abhijeet Dhiman, adhiman@purdue.edu | ${ }^{1}$ School of Aeronautics and Astronautics, Purdue University, 701 W. Stadium Ave, West Lafayette, IN 47907, USA.

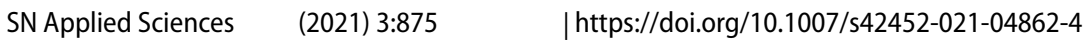


cleavage in $\beta-H M X$ to take place along the $\{011\}$ crystallographic planes.

The ability to understand and, in turn, accurately predict the behavior of EM critically relies, in part, on understanding the elasticity of the material, as the structure and compressibility of the material are relevant regardless of initiation shock mechanics [1]. Description of the anisotropic elastic behavior of $\beta$-HMX has been widely investigated. However, a significant portion of the description has been obtained via molecular dynamics related numerical methods [6]. Experimentally, it has proven difficult to measure elastic properties of energetic crystals such as $\beta$-HMX due to their anisotropic nature requiring complex geometric considerations and their brittle nature yielding relatively minute strains before failure [7]. Previous experimental methods involving elastic constant determination for $\beta$-HMX have involved the use of techniques such as impulsive stimulated light scattering (ISLS) [8], impulsive stimulated thermal scattering (ISTS) [9] and Brillouin scattering [10]. However, these methods, when compared, produce a variance in values that is yet to be fully understood or explained [1]. Nanoindentation as an experimental procedure has also been used in attempts to characterize the hardness and modulus of $\beta-H M X$ in previous studies [5, 11-13]. The results from such studies provide unexplained variance which may only be fully understood with more work in the area.

With respect to EMs, hot spots are regions in the microstructure whereas the initiated volume is sufficiently large, and temperature is sufficiently high to start the chemical reactions leading to detonation. Friction, or interfacial interaction between particles as mentioned above, among other mechanisms, is known to be a cause of hot spot initiation. Susceptibility of an energetic material to such form of failure, most adequately described by the frictional sensitivity of the material, has been shown to be dependent on multiple factors, of which hardness has been speculated to be the most important [14]. In addition to elastic properties, this work also presents a study of the anisotropic hardness values and viscoplasticity parameters of $\beta$-HMX.

Currently, with few exceptions, simulation of the mechanical behavior of HMX-based EMs at the mesoscale generally overlooks the anisotropic properties. However, an argument can be made that incorporation of anisotropic properties of HMX crystals may improve the current ability to predict the energetic behavior of such materials. Examples of such exceptions can be seen in work done by Wang et al. [15], in which a full field method was employed to model the local damage behavior of a HMX-based EM. Other works simulate the anisotropic material behavior HMX to determine anisotropic material responses. Work by Conroy et al. [16] modelled the behavior of $\beta-\mathrm{HMX}$ crystals using first principles density functional theory (DFT). They found that the anisotropic material model predicted experimental results that adequately matched existing data. Zamiri et al. [17] also worked on numerically predicting the anisotropic plastic response of modelled single crystals of HMX based on experimental data. This work focuses on experimentally characterizing anisotropic quasistatic as well as dynamic local constitutive material properties of $\beta-H M X$ crystals using indentation based static and dynamic schemes.

\section{Methods}

This section covers brief explanation of the experimental methods and theories to be used in this work. These methods have been covered in detail in previous works [18-21].

\subsection{Sample preparation}

Untwinned single crystals of $\beta$-HMX were formed by controlled solvent evaporation, at room temperature, from a saturated solution of raw HMX in HPLC grade $99.8 \%$ pure acetone. The solution was mixed at ratio of 0.024:1 by weight of $\mathrm{HMX}$ to acetone respectively and measured into vials with perforated caps for slow evaporation. The descriptions here are of monoclinic single crystal $\beta-H M X$ in space group $\mathrm{P} 2_{1} / n$. with lattice parameters $\mathrm{a}=0.654 \mathrm{~nm}$, $\mathrm{b}=0.736 \mathrm{~nm}, \mathrm{c}=1.104 \mathrm{~nm}$, and $\mathrm{B}=102.66^{\circ}$. Two main morphologies of single crystal were observed, and the respective orientations were identified through $\mathrm{x}$-ray diffraction shown in Fig. 1 below.

The first morphology observed, denoted 'morphology 1 ', exposed facets corresponding to the $\{010\},\{011\}$, and $\{110\}$ crystallographic planes, while the second morphology displayed exposed facets corresponding to $\{011\}$ and $\{110\}$ planes only. In both morphologies, the facets corresponding to the $\{011\}$ planes appeared to be of preferred growth, displaying larger surface areas in most crystals observed. The morphologies observed also grew longitudinally along the 'a' crystallographic axis as shown in Fig. 2. The length distribution (along the preferred a-crystallographic axis) of fully formed crystals varied from $200 \mu \mathrm{m}$ to $4 \mathrm{~mm}$ with an average length of $2 \mathrm{~mm}$ and width (approximately perpendicular to the a-axis) of $1 \mathrm{~mm}$. Typically crystals equal to or larger than $1 \mathrm{~mm}$ were chosen for experiment purposes to provide sufficient surface area for multiple indentations.

Experiments for further characterization of elastic behavior required access to indentation axes perpendicular to planes that were not exposed in the single crystal morphologies that presented, such as the (100) and (001) planes corresponding to the $a$ and $c$ crystallographic 
Fig. 1 Identified faces of $\beta$-HMX crystals for two morphologies: a, c morphology 1 and $\mathbf{b}, \mathbf{d}$ morphology 2
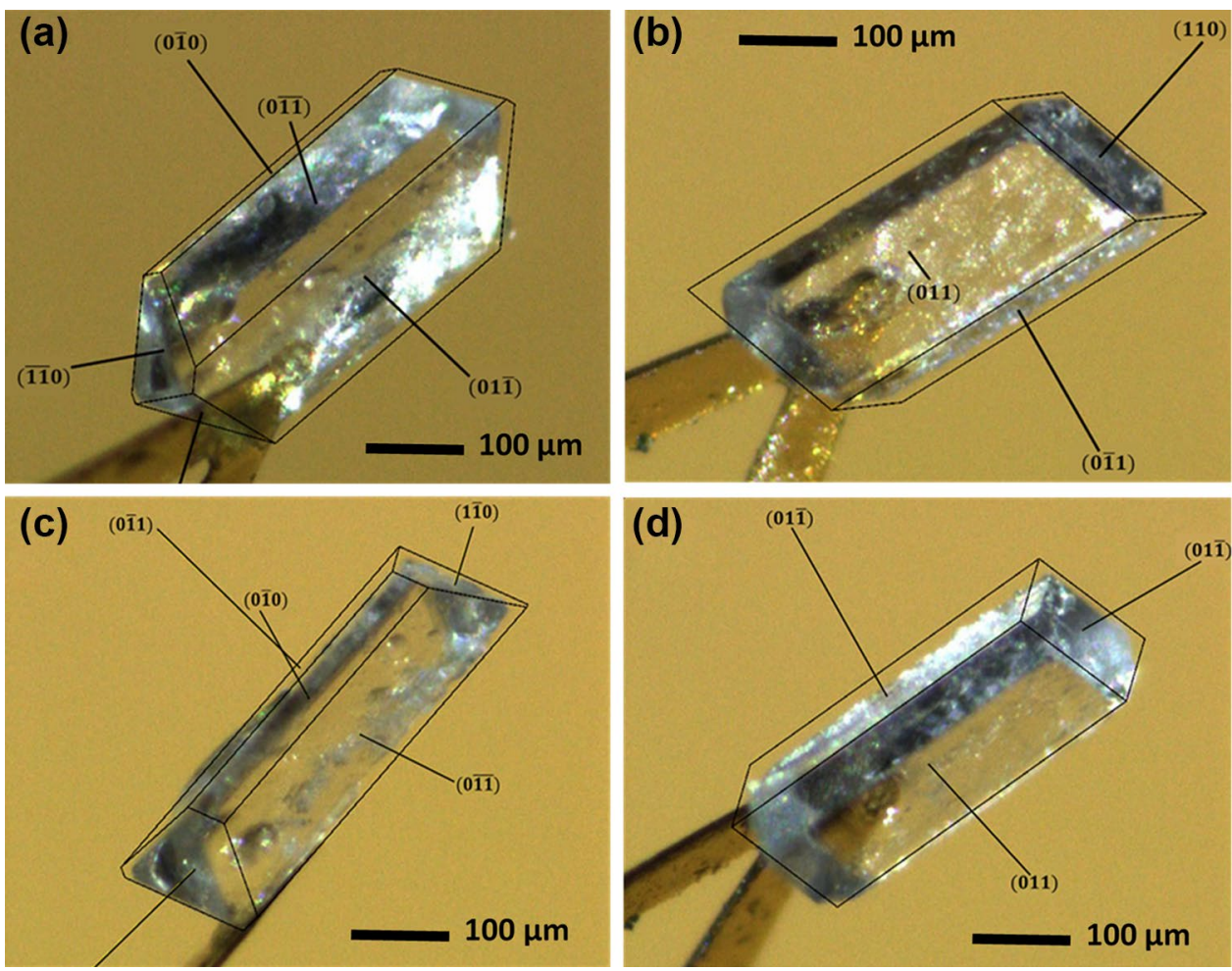

(a)

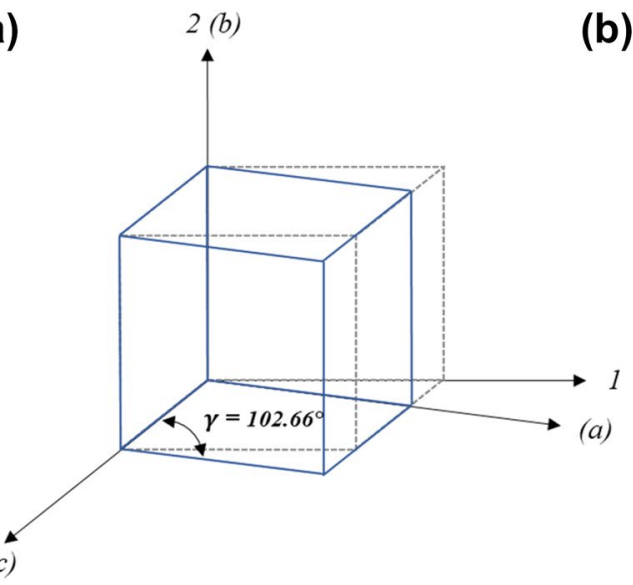

(b)

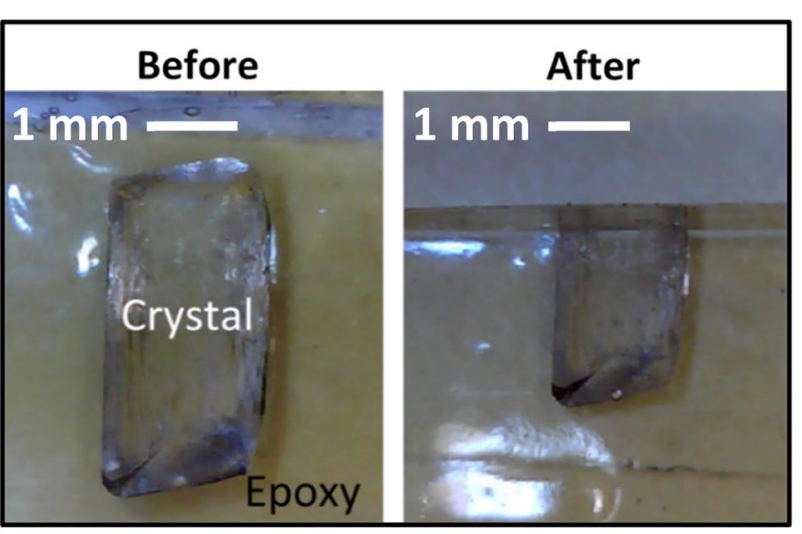

Fig. 2 a Crystallographic axes and planes of monoclinic $\beta$-HMX. b Before and after images showing cleaving of embedded $\beta$-HMX crystal to expose (100) plane

axes. In such cases, crystals were embedded in a hardened epoxy and cleaved to expose the necessary planes and sanded down with decreasing particle sizes. In particular, the hardened epoxy used here was the 'Aluminite Amazing Casting Resin' due to its lower volumetric contraction on curing. The surfaces were then polished using silicon carbide sandpaper with $\mathrm{a}<8 \mu \mathrm{m}$ average particle diameter.

\subsection{Methods to obtain anisotropic elastic constitutive properties}

This work presents experimentally obtained indentation material properties to describe the anisotropic elastic behavior of $\beta-H M X$ as a response to external loading applied to different planes of the crystal. This section describes the experimental and theoretical methods employed. 


\subsubsection{Nanoindentation}

Nanoindentation has been used in multiple studies of material elastic behavior and has proven to be a reliable technique to measure local elastic material properties [21-24], extending to elastic properties of Ems [5]. Properties such as elastic modulus and hardness can be easily obtained via nanoindentation due to the underlying fact that displacements recovered during unloading are known to be mainly elastic. In this work, a NanoTest platform (Micro Materials Ltd., UK) [25] is used to perform the indentation experiments. Multiple indentations were performed, at various indentation depths, on each plane at room temperature with a Berkovich type indenter having a diamond tip radius of $20 \mathrm{~nm}$. Figure 3 a shows the indentation test setup. Sample crystals were oriented with the loading plane of interest perpendicular to the indenter and indentations were spaced with a minimum of $100 \mu \mathrm{m}$ between each indent to limit the possibility of coalescence between indents. The machine compliance is corrected as detailed in previous work [21] (Fig. 3b).

\subsubsection{Hardness and elastic modulus evaluation}

An indentation experiment is characterized by loading applied by the indenter until the desired maximum load is achieved, whereby the maximum load is held for $5 \mathrm{~s}$ and then unloaded. Each indentation experiment produces a load-depth plot, an example of which can be observed in Fig. 4.

The Oliver-Pharr's method [26] is used to calculate the indentation modulus and hardness. In the case of isotropic materials, the Oliver-Pharr method has been shown to estimate material properties such as Young's modulus to a high accuracy, about $4 \%$ of literature values. However, this is not the case for anisotropic materials as the indentation modulus represents more of a weighted average value as

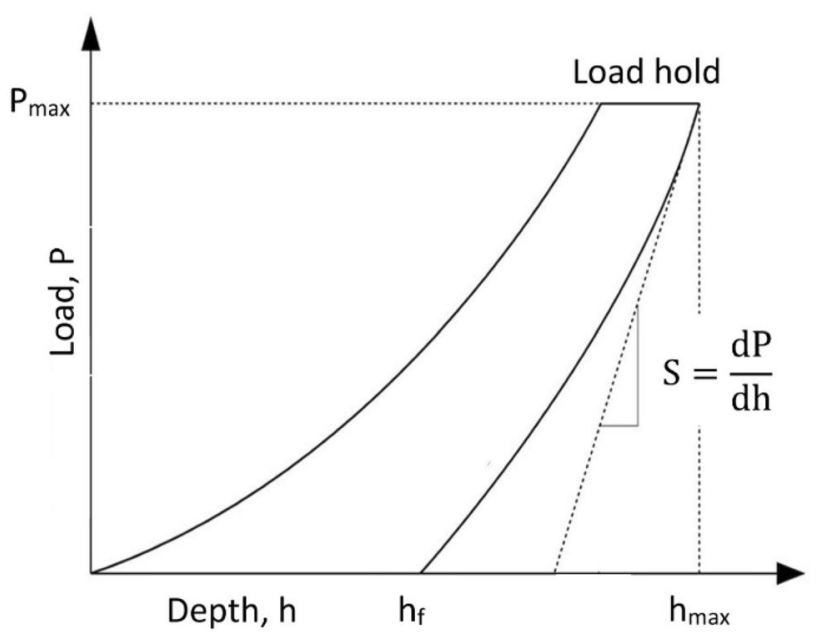

Fig. 4 Indentation load-depth plot

deformation involves multiple directions [27]. Therefore, in this work, the indentation moduli for each direction will be obtained individually, as weighted averages, and compared to actual elastic constants for those directions. To obtain the necessary parameters, the values obtained from the indentation experiment are fit the load-depth curve obtained from the experiment.

$P=A_{m}\left(h-h_{f}\right)^{m}$

where $A_{m}$ and $m$ are parameters obtained empirically from the power law equation, $h$ is the indentation depth corresponding to a specific load $\mathrm{P}$, and $\mathrm{h}_{\mathrm{f}}$ is the plastic indentation depth remaining after the load has been completely removed. The indentation hardness can be calculated as,

$H=\frac{P_{\max }}{A}$ (a)

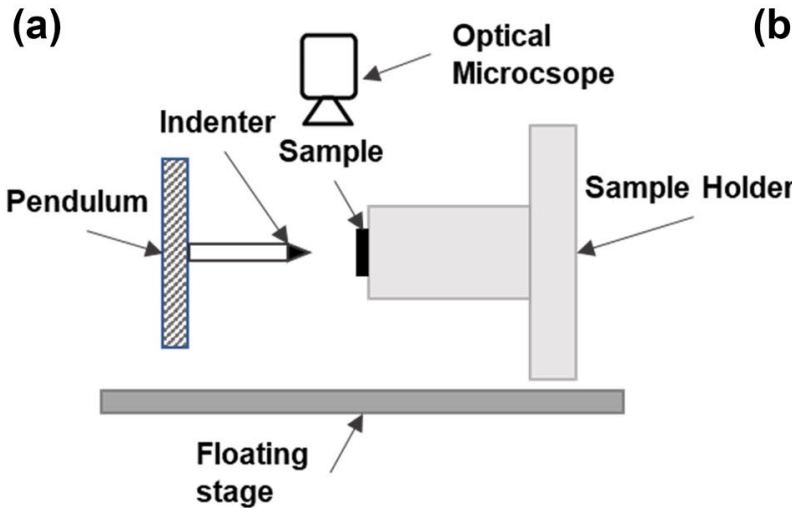

(b)

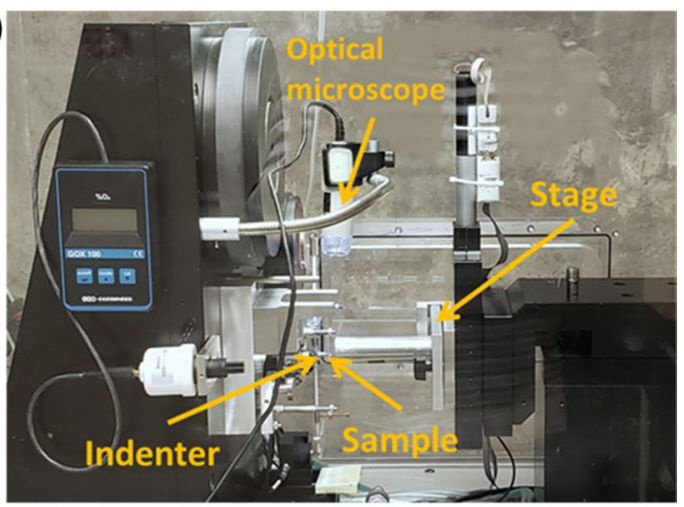

Fig. 3 Nanoindentation experiment setup 
For a Berkovich indenter, the contact area can be estimated as $24.5 h_{c^{\prime}}^{2}$ where $h_{c}$ is the contact depth. The reduced indentation modulus $\left(M_{\mathrm{r}}\right)$ is calculated as,

$M_{r}=\frac{\sqrt{\pi}}{2} \frac{S}{\sqrt{A}}$

The indentation modulus of the material is then obtained from the relation,

$\frac{1}{M_{r}}=\frac{1-v^{2}}{M}+\frac{1-v_{i}^{2}}{E_{i}}$

Constants $\mathrm{U}$ and $\mathrm{M}$ are the Poisson's ratio and indentation modulus of the sample, while $\mathrm{v}_{i}$ and $\mathrm{E}_{\mathrm{i}}$ represents Poisson's ratio and Young's modulus of the indenter, respectively. The Poisson ratios used in this work were obtained from values presented in literature [6] and approximated thus,

$v_{i}=\frac{v_{i j}+v_{i k}}{2}$

Therefore, $0.33,0.3$, and 0.4 were used for $v_{1}, v_{2}, v_{3}$ and respectively.

\subsubsection{Indentation size effect (ISE)}

Previous studies of hardness obtained by nanoindentation have shown a dependence of the measured values of hardness on 'indentation size', i.e. the indirect variation of hardness with indentation depth. This phenomenon is referred to as the indentation size effect (ISE) and some possible explanations of the causes of this behavior, particularly in crystalline materials, have been proposed in literature [28], for instance the strain gradient plasticity theory [21]. The hardness eventually reaches a constant value which can be quantified according to the Nix-Gao relation [28] as:

$\frac{H}{H_{0}}=\sqrt{1+\frac{h^{*}}{h}}$

Here, $\mathrm{H}$ is the hardness value corresponding to a certain indentation depth $\mathrm{h} . \mathrm{H}_{0}$ is the final constant hardness value at infinite depth, and $h^{*}$ is a characteristic length parameter, both of which are important parameters necessary for the definition of constitutive equations in strain gradient plasticity theory [21]. Equation 6 can be rewritten as,

$H^{2}=H_{0}^{2}\left(1+\frac{h^{*}}{h}\right)$
$\mathrm{H}$ and $\mathrm{h}$ values are obtained here from experiment results.

\subsection{Methods to obtain anisotropic strain-rate-dependent viscoplastic constitutive model}

In order to describe the anisotropic viscoplastic behavior of $\beta$-HMX crystals, a viscoplastic constitutive power law is defined, and experimental orientation-dependent viscoplastic parameters are obtained in this work. In a previous work [29], the behavior of Hydroxy-Terminated Polybutadiene (HTPB)-HMX based PBXs have been modelled using a cohesive finite element method (CFEM) based simulation software. The developed CFEM model used polycrystalline HMX parameters in order to obtain an approximated description of the viscoplastic behavior of the material. Here, we present a study into the orientation dependence of these parameters, giving a more detailed description of the range of possible behavioral limits and how well previous approximations fit into this range.

\subsubsection{Dynamic small-scale impact experiments}

The viscoplastic constitutive parameters are obtained via nanoscale dynamic impact in regions of interest on selected $\beta$-HMX crystal phases. The specific equipment used for this experiment is impact module of Micro Materials, UK. This equipment and the corresponding technique have been used to successfully obtain mechanical properties of other EMs at small scale such as HTPB-AP interface [30]. In this experimental setup, the sample is positioned in front of a spherical impactor with the plane face of interest oriented perpendicular to the impactor. The sample is mounted on a three-dimensional stage and the entire setup sits on a "floating" platform; thus, the sample can be moved freely in all directions. The impactor is a conical indenter of radius $1 \mu \mathrm{m}$, it is mounted on a vertical pendulum which hangs on a frictionless spring. Impact on the sample is achieved through the use of electromagnets on the vertical pendulum and as such different magnitudes of desired impact force can be achieved. The schematic for the equipment setup is shown in Fig. 5 , [19], below.

Depth is measured by the distance between the capacitors located behind the impactor and the velocity is obtained by a continuous differentiation of the depthtime plot. Impact is performed on each plane of interest on multiple samples. The stress, strain, and average strain rate of the experiment can be obtained using values from the depth-time and velocity-time plot. Figure $5 \mathrm{~b}$ shows an example of a plot output from one impact experiment. The impact strain rate is calculated as, 


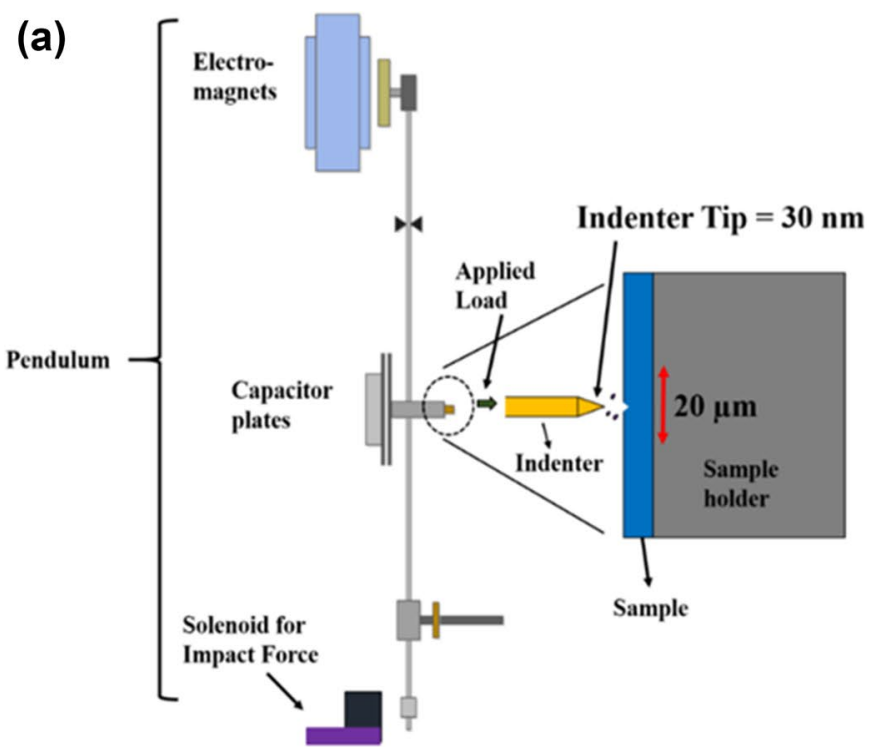

(b)

Fig. 5 a Small-scale dynamic impact experiment setup. b Load-depth plot and derived velocity-time plot from one impact experiment

$$
\dot{\varepsilon} \approx \frac{V_{\text {in }}}{h_{\text {max }}}
$$

where $\mathrm{h}_{\max }$ is the maximum depth and $\mathrm{V}_{\text {in }}$ is the maximum velocity. The strain and stress are also calculated as,

$\varepsilon \approx \frac{h_{r e s}^{2}}{h_{\max }^{2}}$

$\sigma \approx \frac{P}{\pi h_{\max }^{2}}$

where $h_{\text {res }}$ is the residual depth and $P$ is the impact load. These values are fit to a power law model which is assumed to represent the effective stress-effective viscoplastic strain relationship.

\subsubsection{Viscoplastic constitutive parameter evaluation}

Following the method outlined by Prakash et al. [18], we obtain the orientation dependent viscoplastic model parameters for each plane by fitting the load related values obtained above to a power law model:

$\bar{\sigma}=A\left(\bar{\varepsilon}^{v p}\right)^{n}$,

where

$A=F_{0}\left(\frac{\dot{\bar{\varepsilon}}^{v p}}{\dot{\bar{\varepsilon}}^{0}}\right)^{m}$.
Here, $\bar{\varepsilon}^{v p}$ is the effective viscoplastic strain, and $\dot{\bar{\varepsilon}}^{v p}$ is the effective viscoplastic strain rate. The coefficient, $A$, is plotted against the effective viscoplastic strain rate on a log-log scale to obtain $F_{0}$ and m parameters more simply as the slope and intercept of the plot. The parameters fit to a power law model useful for predicting the constitutive behavior of the material at higher strain rates [30]. Here, the parameter ' $m$ ' represents the strain rate exponent and the parameter ' $n$ ' represents the strain hardening exponent, signifying the quantifiable influence of the effective viscoplastic strain rate and strain respectively on the measured effective stress. The validity of the viscoplastic equivalent stress-strain power law for modeling the behavior of this material has been evaluated in previous work [18]. Here, we obtain the parameters to define the anisotropic viscoplastic behavior in each different orientations of interest in the examined single crystal.

\section{Results and discussion}

Using the process detailed above, at different loads, multiple indentations were performed on each plane of interest on the HMX crystals. Grown crystal samples presented smooth surfaces parallel to $\{010\},\{011\}$, and $\{110\}$ planes and these were used in experiments without modification. Indentations were also performed on planes perpendicular to the three crystallographic axes to characterize behavior on these planes. Two out of three of these planes, (100) and (001) perpendicular to the monoclinic $a$ and $c$ axes, respectively, did not 


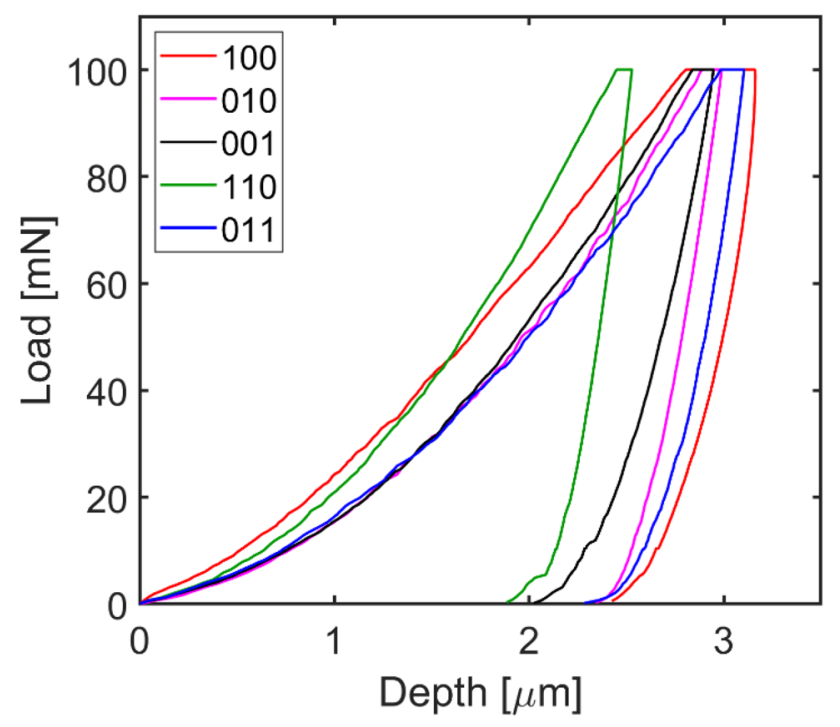

Fig. 6 Indentation load versus depth plot comparisons of different planes of $\beta$-HMX crystal

present parallel facets naturally in the samples grown. As such, modifications, detailed above (Fig. 2), were made to expose them. The plane perpendicular to the $b$ crystallographic axis, the (010) plane, naturally presented on examined crystal samples as shown in Fig. 1 earlier (Fig. 6).
In order to define the indentation moduli for the three main axial directions $(\{010\},\{011\}$, and $\{110\})$, an additional plane was required to be exposed for loading. Indentation moduli corresponding to the elastic moduli, $\mathrm{E}_{22}$ and $\mathrm{E}_{33}$, were obtained directly as the $b$ and $c$ crystallographic axes were intentionally oriented along the 2 and 3 axes as shown in Fig. 2a. The indentation modulus corresponding to the directional elastic modulus, $\mathrm{E}_{11}$, however, were obtained by loading along a direction determined by the cross product between the $a$ and $c$ crystallographic axes. The results of the moduli obtained by indentation for these three directions are presented in Table 1 (Fig. 7).

Our results for modulus in the $\mathrm{E}_{22}$ direction showed good agreement with the value presented by Kucheyev et al. [12], in which Berkovich indentations were performed. The work of Li et al. [11] also presents moduli for different facets of $\beta$-HMX crystals as obtained by Berkovich indentation. However, our values are significantly lower than their presented results. As discussed above, this is not unique to nanoindentation as other means of experimental determination of elastic moduli for this material also present discrepancies. It should be noted however, that values obtained present a similarity to values obtained by molecular dynamics simulations [31] with a maximum of $12 \%$ error, and otherwise reasonably fit into the statistically determined range of values presented, obtained from both numerical and experimental methods. Moreover,

Table 1 Indentation moduli obtained from this work in comparison to elastic moduli presented in published works

\begin{tabular}{lllllll}
\hline$E_{\text {ii }}$ & This work & Li et al. [11] & $\begin{array}{l}\text { Kucheyev } \\
\text { et al. [12] }\end{array}$ & $\begin{array}{l}\text { Stevens } \\
\text { et al. [10] }\end{array}$ & Zaug et al. [8] & Sewell et al. [6] \\
\hline $\mathrm{E}_{11}$ & 14.21 & - & - & 8.58 & 11.82 & 14.23 \\
$\mathrm{E}_{22}$ & 16.65 & 23.18 & 17.6 & 10.95 & 24.04 & 14.88 \\
$\mathrm{E}_{33}$ & 11.65 & 26.05 & - & 5.37 & 9.69 & 12.85 \\
\hline
\end{tabular}

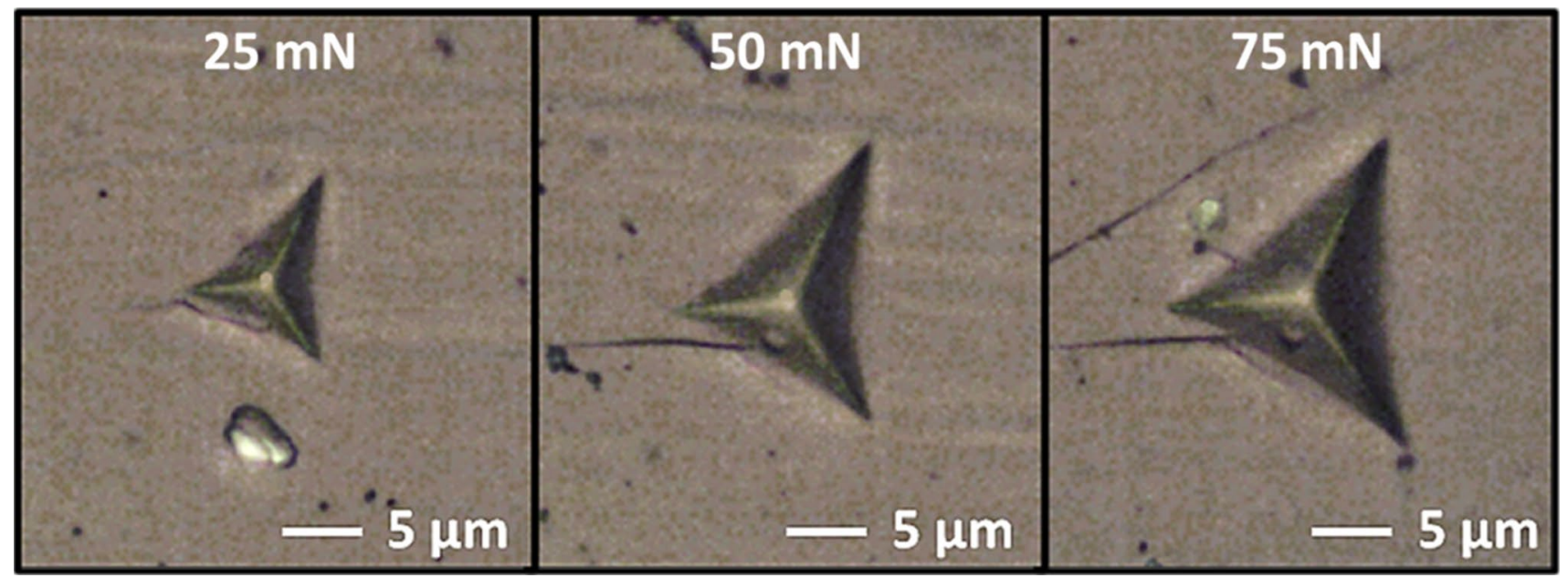

Fig. 7 Imaged indentations performed on the (010) facet of a $\beta$-HMX crystal 

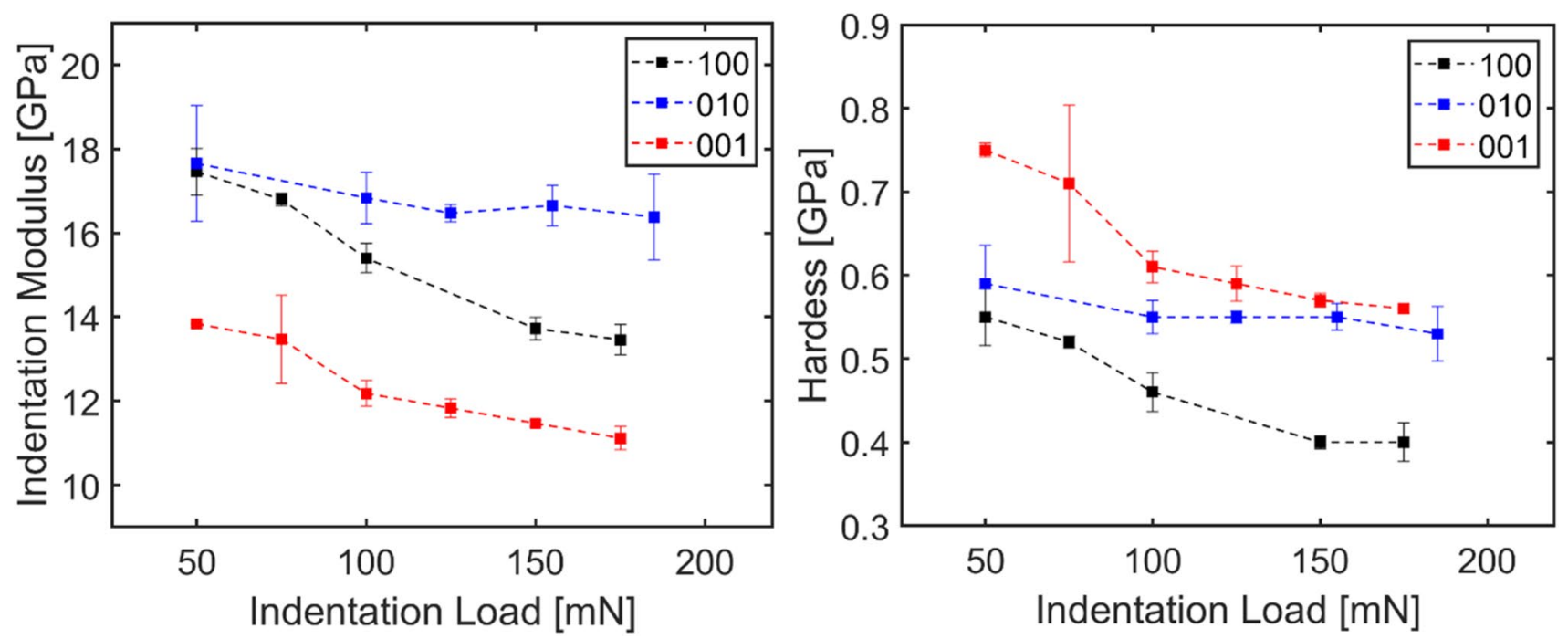

Fig. 8 Anisotropic behavior of $\beta$-HMX crystal planes with respect to modulus and hardness

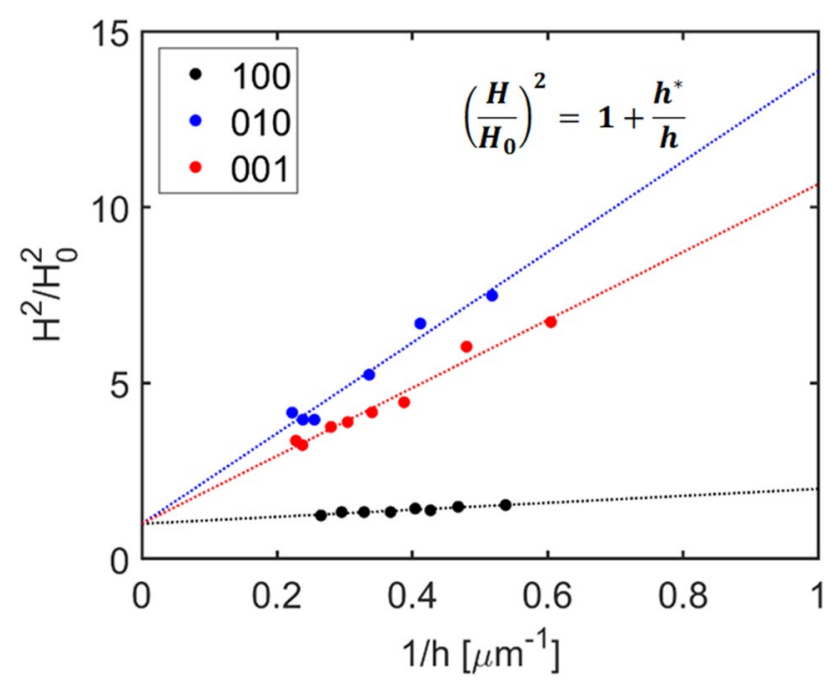

Fig. 9 Comparisons of Nix Gao fitting of hardness data for 3 planes

the values obtained in this work agree with most works which show $E_{22}$ to be the largest and $E_{33}$ to be the smallest moduli.

The anisotropic behavior of three different orientations with respect to indentation modulus and hardness can be observed in Fig. 8. Here, the properties of planes corresponding to the three main crystallographic axes are compared and anisotropic behavior can clearly be observed in the values of measured properties with changing indentation load/depth. The hardness and plastic depth values were fit using the Nix-Gao relation detailed above, the results are shown in Fig. 9. The indentation moduli, hardness, and Nix-Gao relation fitted values obtained for each plane are presented in Table 2. Li et al. [11] presented
Table 2 Properties obtained for each tested plane of $\beta$-HMX by indentation

\begin{tabular}{llllr}
\hline Plane & $\mathrm{M}(\mathrm{GPa})$ & $\mathrm{H}(\mathrm{GPa})$ & $\mathrm{H}_{0}(\mathrm{GPa})$ & $\mathrm{h}^{*}(\mu \mathrm{m})$ \\
\hline 100 & $14.84 \pm 0.33$ & $0.42 \pm 0.02$ & 0.20 & 12.88 \\
010 & $16.65 \pm 0.57$ & $0.55 \pm 0.02$ & 0.48 & 0.99 \\
001 & $11.65 \pm 0.22$ & $0.56 \pm 0.01$ & 0.29 & 9.66 \\
110 & $21.04 \pm 0.59$ & $0.65 \pm 0.02$ & 0.56 & 127.55 \\
011 & $14.79 \pm 0.38$ & $0.51 \pm 0.01$ & 0.26 & 8.97 \\
\hline
\end{tabular}

hardness values for the (010) face of $\beta-\mathrm{HMX}$ as $1.13 \mathrm{GPa}$, also obtained via Berkovich indentations on the crystal facet. This is in general agreement with the value of $1 \mathrm{GPa}$, for arbitrarily oriented HMX crystals, presented by Burch et al. [13], however our value of $0.55 \mathrm{GPa}$ for the same facet (010) are significantly lower and closer to the value of 0.65 GPa presented by Kucheyev et al. [12].

A significant amount of the data on hardness for $\beta-H M X$ presented in literature focuses on the Vickers Hardness Number (VHN). Studies have shown the hardness values from Vickers indentations to be similar to, however slightly lower than, values obtained for Berkovich indentations $[32,33]$. The hardness values obtained here are higher but close in value to the VHNs; $0.41 \mathrm{GPa}$ obtained by Amuzu et al. [34] and 0.4 GPa obtained by Palmer and Field [5]. However, both works did not indicate specific orientations for the values. A VHN range of $0.3-0.36 \mathrm{GPa}$, presented by Gallagher et al. [2] for indentations made on the (010), $\{011\}$, and $\{110\}$ planes, fall lower comparatively.

Viscoplastic parameters were also obtained in this work for the viscoplastic effective stress-strain power law described above. These parameters were obtained via 
small-scale dynamic impact experiments, to describe the viscoplastic behavior. Viscoplastic parameters for polycrystalline HMX embedded in a polymeric binder have been obtained in a previous work [35]. As the material was polycrystalline, due to the underlying assumption of isotropy, the parameters obtained were independent of orientation. The parameters for Ammonium Perchlorate (AP) have also been obtained in a previous work and both parameters are included below for comparison. The orientation dependent viscoplastic parameters for the viscoplastic effective stress-strain power law are presented in Table 3. Figure 10 shows the fitting of the data obtained for the (100) plane to the viscoplastic power law at multiple strain rates.

From the results presented above, we observed that viscoplastic strain for the $\{110\}$ plane appears to have the highest comparative dependency on strain rate, as determined by the stain rate exponent ' $m$ '. The strain values are predicted to be the highest in comparison

Table 3 Anisotropic viscoplastic parameters

\begin{tabular}{llll}
\hline Plane & $F_{0}$ & $\mathrm{~m}$ & $\mathrm{n}$ \\
\hline 100 & 1.06 & 0.13 & 0.26 \\
010 & 0.46 & 1.17 & 0.33 \\
001 & 0.32 & 1.8 & 0.16 \\
110 & 0.11 & 2.1 & 0.59 \\
011 & 0.21 & 1.83 & 0.27 \\
AP $^{\mathrm{a}}$ & 0.016 & 1.61 & 0.17 \\
Polycrysta $^{\mathrm{b}}$ & 0.95 & 0.5 & 0.63 \\
\hline
\end{tabular}

a[18]

${ }^{b}[35]$ to other planes at a given strain rate and stress. This behavior is as expected as this plane has a high value of the strain hardening exponent ' $n$ ' as well. These findings appear to agree with results obtained by Zamiri et al. [17] in the anisotropic simulation of $\beta-H M X$, where simulation, and compared experimental data [7], showed the $\{110\}$ plane to be relatively more prone to plastic deformation. In addition, the results potentially corroborate work by Conroy et al. [16] which found maximum principal stress to be greatest for the $\{110\}$ and $\{011\}$ plane compressions. The (100) plane appears to have the lowest dependency on strain rate and resultingly low comparative viscoplastic strains. Viscoplastic behavior of AP will be closer to the behavior predicted for most planes in terms of strain rate dependency, as the strain rate exponent value is closer to the average. However, in comparison to most planes, the values for polycrystalline HMX predict low viscoplastic strains closer to the lowest values of the (100) plane, with similar exponent values. Figure 11 shows the comparative values of the exponent parameters.

An interesting finding in this work is that the planes with the most and least comparative viscoplastic strains also had the highest and lowest hardness, respectively. This result is considered to be as expected as materials with higher hardness values will in turn have a higher values of yield strength.
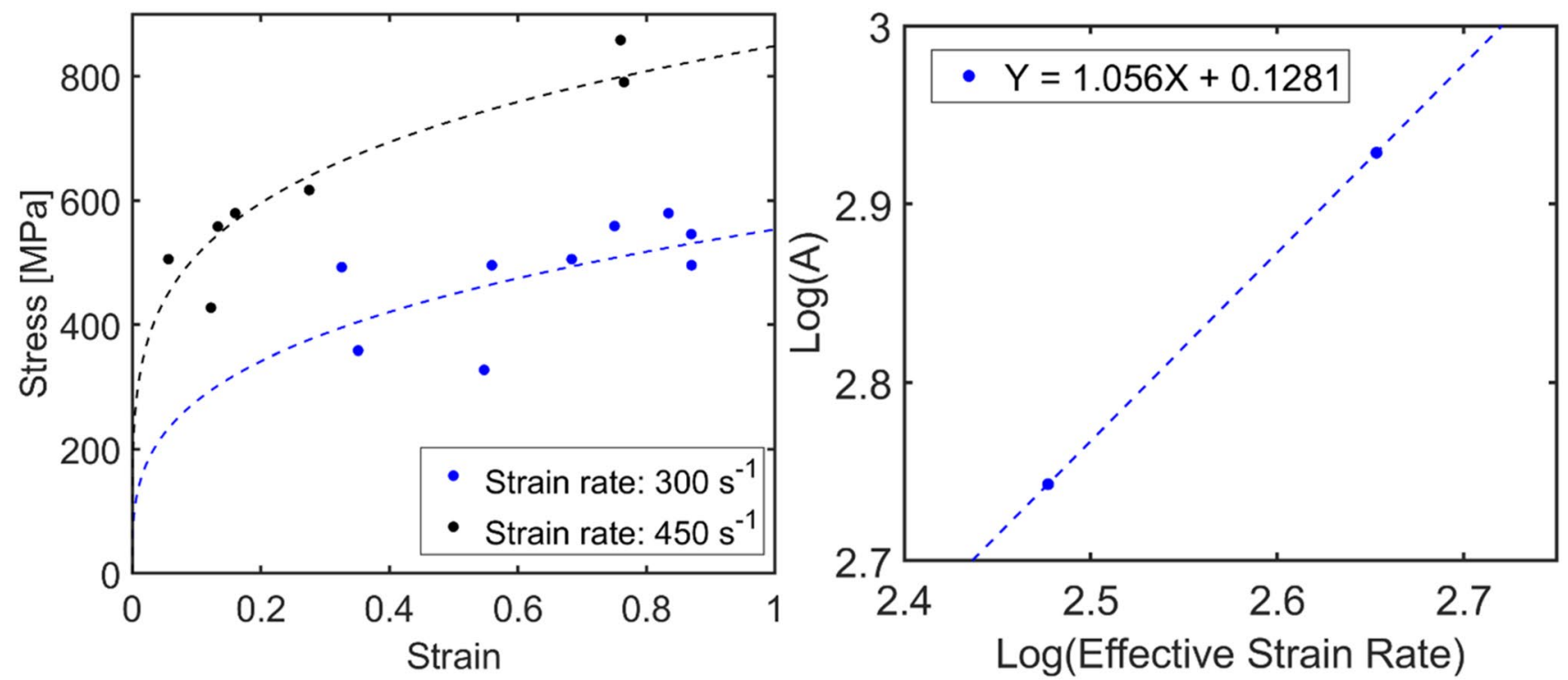

Fig. 10 Viscoplastic model parameters are obtained by fitting impact data to viscoplastic power law from 100 plane 


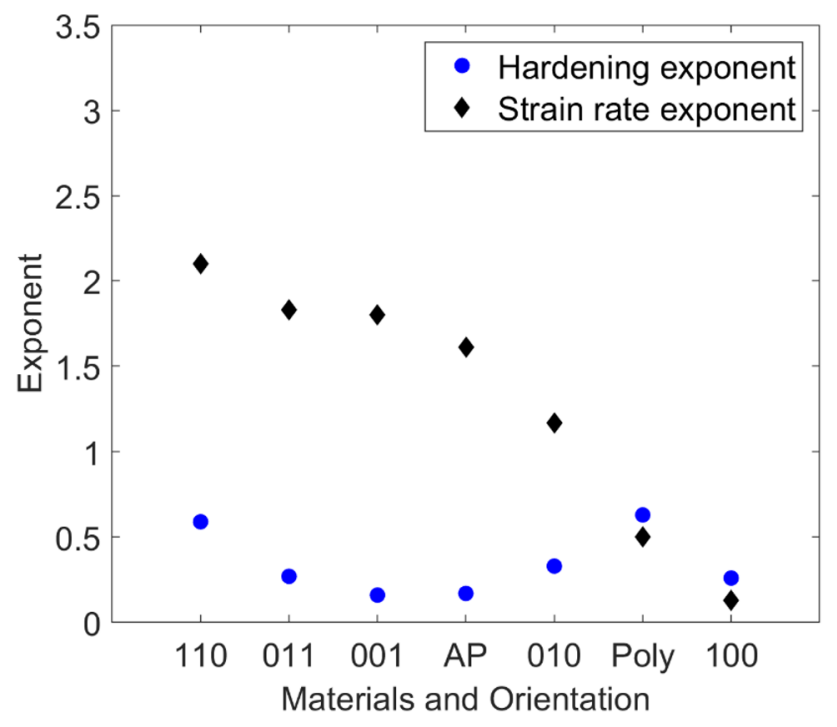

Fig. 11 Comparisons of exponent values in viscoplastic model

\section{Conclusions}

In this work, experimentally obtained constitutive parameters for modeling the anisotropy dependent mechanical behavior of $\beta-H M X$ were presented. Indentation modulus and hardness values were obtained for each plane via nanoindentation experiments. Results obtained fit well into a statistically determined range of values obtained from both numerical and experimental methods presented in literature. Nanoindentation has been shown to estimate the elastic modulus of isotropic materials to a 4\% accuracy and as such, these results and future validations may present a discussion on the possibility of nanoindentation providing a means of obtaining acceptable approximations of anisotropic elastic moduli.

Viscoplastic constitutive parameters were also obtained for each plane by fitting the values obtained from dynamic impact experiments to a power law model. The results showed the $\{110\}$ plane to have the highest comparative viscoplastic strains respectively at a given strain rate and stress, which is supported by other findings in literature. The parameters presented in this work could provide a more accurate description of the anisotropic behavior of $\beta-\mathrm{HMX}$ in the prediction of the performance of HMX based EMs.

Funding This study was funded by the US Air Force Office of Scientific Research (Grant Number: FA9550-19-1-0318, program manager: Dr. Martin Schmidt).

\section{SN Applied Sciences}

\section{Declarations}

Conflict of interest The authors declare that they have no conflict of interest.

Open Access This article is licensed under a Creative Commons Attribution 4.0 International License, which permits use, sharing, adaptation, distribution and reproduction in any medium or format, as long as you give appropriate credit to the original author(s) and the source, provide a link to the Creative Commons licence, and indicate if changes were made. The images or other third party material in this article are included in the article's Creative Commons licence, unless indicated otherwise in a credit line to the material. If material is not included in the article's Creative Commons licence and your intended use is not permitted by statutory regulation or exceeds the permitted use, you will need to obtain permission directly from the copyright holder. To view a copy of this licence, visit http://creativecommons. org/licenses/by/4.0/.

\section{References}

1. Hooks DE, Ramos KJ, Bolme CA, Cawkwell MJ (2015) Elasticity of crystalline molecular explosives. Propellants Explos Pyrotech 40:333-350. https://doi.org/10.1002/prep.201400282

2. Gallagher HG, Sherwood JN, Vrcelj RM (2014) Growth and dislocation studies of $\beta$-HMX. Chem Cent J 8:75. https://doi.org/10. 1186/s13065-014-0075-y

3. Baer MR (2002) Modeling heterogeneous energetic materials at the mesoscale. Thermochim Acta 384:351-367. https://doi.org/ 10.1016/S0040-6031(01)00794-8

4. Palmer SJP, Field JE, Huntley JM (1993) Deformations, strength and strains to failure of polymer bonded explosives. Proc R Soc Lond Ser A Math Phys Sci 440:399-419

5. Palmer SJP, Field JE (1982) The deformation and fracture of $\beta$-HMX. Proc R Soc Lond Ser A Math Phys Sci 383:399-407

6. Sewell TD, Menikoff R, Bedrov D, Smith GD (2003) A molecular dynamics simulation study of elastic properties of HMX. J Chem Phys 119:7417-7426. https://doi.org/10.1063/1.1599273

7. Rae PJ, Hooks D, Liu C (2006) The stress versus strain response of single $\beta-H M X$ crystals in quasi-static compression. In: Proceedings of the 13th international detonation symposium, IDS 2006, pp 293-301

8. Zaug JM (1998) Elastic constants of B-HMX and tantalum, equations of state of supercritical fluids and fluid mixtures and thermal transport determinations, United States

9. Sun B, Winey JM, Gupta YM, Hooks DE (2009) Determination of second-order elastic constants of cyclotetramethylene tetranitramine $(\beta-H M X)$ using impulsive stimulated thermal scattering. J Appl Phys 106:053505. https://doi.org/10.1063/1.3211927

10. Stevens LL, Eckhardt CJ (2005) The elastic constants and related properties of $\beta-H M X$ determined by Brillouin scattering. J Chem Phys 122:174701. https://doi.org/10.1063/1.1883627

11. Li M, Tan W-J, Kang B, Xu R-J, Tang W (2010) The elastic modulus of $\beta$-HMX crystals determined by nanoindentation. Propellants Explos Pyrotech 35:379-383. https://doi.org/10.1002/prep. 201000018

12. Kucheyev SO, Gash AE, Lorenz T (2014) Deformation and fracture of LLM-105 molecular crystals studied by nanoindentation. Mater Res Express 1:025036. https://doi.org/10.1088/20531591/1/2/025036

13. Burch A, Yeager J, Bahr D (2017) Nanoindentation of HMX and idoxuridine to determine mechanical similarity. Curr ComputAided Drug Des. https://doi.org/10.3390/cryst7110335 
14. Harris J, United S, National Technical Information S (1982) Friction sensitivity of primary explosives. U.S. Dept. of Commerce, National Technical Information Service, Springfield, Va

15. Wang $X$, Wu $Y$, Huang $F(2017)$ Numerical mesoscopic investigations of dynamic damage and failure mechanisms of polymer bonded explosives. Int J Solids Struct 129:28-39. https://doi.org/ 10.1016/j.ijsolstr.2017.09.017

16. Conroy MW, Oleynik II, Zybin SV, White CT (2008) First-principles anisotropic constitutive relationships in $\beta$-cyclotetramethylene tetranitramine ( $\beta$-HMX). J Appl Phys 104:053506. https://doi.org/ $10.1063 / 1.2973689$

17. Zamiri A, De $S$ (2011) Modeling the anisotropic deformation response of $\beta$-HMX molecular crystals. Propellants Explos Pyrotech. https://doi.org/10.1002/prep.201000037

18. Prakash C, Emre Gunduz I, Tomar V (2018) Effect of strain rate and interface chemistry on failure in energetic materials. In: Carroll J, Xia S, Beese AM, Berke RB, Pataky GJ (eds) Fracture, fatigue, failure and damage evolution, vol 7. Springer, Cham, pp 7-12

19. Prakash C, Gunduz IE, Oskay C, Tomar V (2018) Effect of interface chemistry and strain rate on particle-matrix delamination in an energetic material. Eng Fract Mech 191:46-64. https://doi.org/ 10.1016/j.engfracmech.2018.01.010

20. Olokun A, Prakash C, Emre Gunduz I, Tomar V (2019) Interface chemistry dependent mechanical properties in energetic materials using nano-scale impact experiment. In: Kimberley J, Lamberson LE, Mates S (eds) Dynamic behavior of materials, vol 1. Springer, Cham, pp 147-152

21. Wang H, Dhiman A, Ostergaard HE, Zhang Y, Siegmund T, Kruzic JJ, Tomar V (2019) Nanoindentation based properties of Inconel 718 at elevated temperatures: a comparison of conventional versus additively manufactured samples. Int J Plast 120:380394. https://doi.org/10.1016/j.ijplas.2019.04.018

22. Marsh J, Han YS, Verma D, Tomar V (2015) An investigation into plastic deformation of irradiated tungsten microstructure at elevated temperatures using the Anand's viscoplastic model. Int J Plast 74:127-140

23. Wang H, Thomas J, Okuniewski MA, Tomar V (2020) Constitutive modeling of $\delta$-phase zircaloy hydride based on strain rate dependent nanoindentation and nano-scale impact dataset. Int J Plast 133:12787

24. Zhang Y, Mohanty DP, Seiler P, Siegmund T, Kruzic JJ, Tomar V (2017) High temperature indentation based property measurements of IN-617. Int J Plast 96:264-281

25. Verma D, Tomar V (2014) Structural-nanomechanical property correlation of shallow water shrimp (Pandalus platyceros) exoskeleton at elevated temperature. J Bionic Eng 11:360-370. https://doi.org/10.1016/S1672-6529(14)60049-4
26. Oliver WC, Pharr GM (1992) An improved technique for determining hardness and elastic modulus using load and displacement sensing indentation experiments. J Mater Res 7:15641583. https://doi.org/10.1557/JMR.1992.1564

27. Fan Z, Swadener JG, Rho JY, Roy ME, Pharr GM (2002) Anisotropic properties of human tibial cortical bone as measured by nanoindentation. J Orthop Res 20:806-810. https://doi.org/10. 1016/S0736-0266(01)00186-3

28. Nix WD, Gao H (1998) Indentation size effects in crystalline materials: a law for strain gradient plasticity. J Mech Phys Solids 46:411-425. https://doi.org/10.1016/S0022-5096(97)00086-0

29. Olokun AM, Prakash C, Emre Gunduz I, Tomar V (2020) The role of microstructure in the impact induced temperature rise in hydroxyl terminated polybutadiene (HTPB)-cyclotetramethylene-tetranitramine (HMX) energetic materials using the cohesive finite element method. J Appl Phys 128:065901. https://doi. org/10.1063/5.0011264

30. Prakash C, Olokun A, Emre Gunduz I, Tomar V (2019). In: Bhattacharya S, Agarwal AK, Rajagopalan T, Patel VK (eds) Nanoenergetic materials. Springer, Singapore, pp 275-290. https:// doi.org/10.1007/978-981-13-3269-2_13

31. Pereverzev A, Sewell T (2020) Elastic coefficients of $\beta-H M X$ as functions of pressure and temperature from molecular dynamics. Curr Comput-Aided Drug Des. https://doi.org/10.3390/cryst 10121123

32. Sakharova NA, Fernandes JV, Antunes JM, Oliveira MC (2009) Comparison between Berkovich, Vickers and conical indentation tests: a three-dimensional numerical simulation study. Int J Solids Struct 46:1095-1104. https://doi.org/10.1016/j.ijsolstr. 2008.10.032

33. Leitner A, Maier-Kiener V, Kiener D (2017) Extraction of flow behavior and Hall-Petch parameters using a nanoindentation multiple sharp tip approach. Adv Eng Mater 19:1600669. https:// doi.org/10.1002/adem.201600669

34. Amuzu JKA, Briscoe BJ, Chaudhri MM (1976) Frictional properties of explosives. J Phys D Appl Phys 9:677-677. https://doi.org/ 10.1088/0022-3727/9/4/518

35. Prakash C, Olokun A, Gunduz IE, Tomar V (2019). In: Bhattacharya S, Agarwal AK, Rajagopalan T, Patel VK (eds) Energy, environment, and sustainability. Springer, Singapore

Publisher's Note Springer Nature remains neutral with regard to jurisdictional claims in published maps and institutional affiliations. 